\title{
Self-similar solutions of semilinear wave equations with a focusing nonlinearity
}

\author{
Piotr Bizoń $^{1}$, Dieter Maison ${ }^{2}$ and Arthur Wasserman ${ }^{3}$ \\ ${ }^{1}$ Institute of Physics, Jagiellonian University, Kraków, Poland \\ 2 Max-Planck-Institute für Physik, Werner-Heisenberg-Institut, Munich, Germany \\ ${ }^{3}$ Department of Mathematics, University of Michigan, Ann Arbor, MI, USA
}

Received 6 February 2007, in final form 4 July 2007

Published 25 July 2007

Online at stacks.iop.org/Non/20/2061

Recommended by F Merle

\begin{abstract}
We prove that in three space dimensions a nonlinear wave equation $u_{t t}-\Delta u=$ $u^{p}$, with $p \geqslant 7$ being an odd integer, has a countable family of regular spherically symmetric self-similar solutions.
\end{abstract}

Mathematics Subject Classification: 35L70, 34B15

\section{Introduction}

An important feature of many nonlinear wave equations is that their solutions, corresponding to smooth initial data, may form singularities after a finite time. Such a phenomenon, usually referred to as blowup, has been a subject of intensive studies beginning with the pioneering works by Keller [1], John [2] and Glassey [3] (we refer the interested reader to the excellent online review of this subject with the complete bibliography [4]).

In this paper we consider the semilinear wave equation with a power nonlinearity

$$
\Phi_{t t}-\Delta \Phi-\Phi^{p}=0, \quad \Phi=\Phi(t, x), \quad x \in R^{3},
$$

where $p \geqslant 7$ is an odd integer. The sign of the nonlinear term corresponds to focusing which means that it tends to magnify the amplitude of the wave. If $\Phi$ is small this term is negligible and the evolution is essentially linear, leading to dispersion. However, if $\Phi$ is large the dispersive effect of the Laplacian may be overcome by the focusing effect of the nonlinearity and a singularity can form. Actually, neglecting the Laplacian altogether and solving the ordinary differential equation $\Phi_{t t}=\Phi^{p}$ one gets the exact, homogeneous in space, solution

$$
\Phi_{0}=\frac{b_{0}}{(T-t)^{\alpha}}, \quad b_{0}=\left[\frac{2(p+1)}{(p-1)^{2}}\right]^{\frac{1}{p-1}}, \quad \alpha=\frac{2}{p-1}, \quad T>0,
$$

which blows up as $t \rightarrow T$. By the finite speed of propagation one can truncate this solution in space to get a solution with compactly supported initial data which blows up in finite time. 
There is theoretical [5] and numerical [6] evidence that the solution $\Phi_{0}$ determines the leading order asymptotics of blowup for generic large initial data. However, for specially prepared initial data, in particular for data fine-tuned to the threshold for blowup, singularities may have a different form which is given by self-similar solutions of equation (1). Such solutions were found numerically in [6]. The aim of this paper is to give a rigorous proof of their existence and discuss their properties.

By definition, self-similar solutions are invariant under rescaling

$$
\Phi(t, x) \rightarrow \Phi_{\lambda}(t, x)=\lambda^{-\alpha} \Phi(t / \lambda, x / \lambda),
$$

hence in the spherically symmetric case they have the form

$$
\Phi(t, r)=(T-t)^{-\alpha} u(\rho), \quad \rho=\frac{r}{T-t},
$$

where $T$ is a positive constant. Note that each self-similar solution, if it is regular for $t<T$, provides an explicit example of regular initial data developing a singularity in a finite time. Substituting the ansatz (4) into equation (1) we obtain the ordinary differential equation for the similarity profile $u(\rho)$

$$
\left(1-\rho^{2}\right) u^{\prime \prime}+\left(\frac{2}{\rho}-(2+2 \alpha) \rho\right) u^{\prime}-\alpha(\alpha+1) u+u^{p}=0 .
$$

It is easy to see that this equation has the constant solution $u_{0}(\rho)=b_{0}$ which, of course, corresponds to the homogeneous solution $\Phi_{0}$ of equation (1). As mentioned above, numerical results indicate that, besides $u_{0}$, there also exist nontrivial regular solutions of equation (5). We remark that equation (5) has been studied by Kavian and Weissler [7] who made many interesting observations about the behaviour of solutions but unfortunately they imposed very restrictive fall-off conditions at infinity which excluded nontrivial solutions. However, as long as one wants to have an example of blowup, due to the finite speed of propagation, only the behaviour of solutions inside the past light cone of the blowup point $(t=T, r=0)$ is relevant, which corresponds to the interval $0 \leqslant \rho \leqslant 1$. As we shall see below regular selfsimilar solutions are parametrized by their values at the endpoints $c=u(0)$ and $b=u(1)$. Due to the singular nature of equation (5) at $\rho=0$ and $\rho=1$, the generic solution obeying the regularity condition at $\rho=0$ becomes singular at $\rho=1$ and vice versa; hence globally regular solutions can exist only for discrete values of the parameters $b$ and $c$. The goal of this paper is to show that there exists an infinite sequence of pairs $\left(b_{n}, c_{n}\right)$ which give rise to globally regular solutions. To this end, in section 2 we first prove the existence of local solutions near the endpoints. Then, in section 3, we show, using a shooting argument, that for a discrete set of values of the parameters these local solutions match smoothly at a midpoint. In section 4 we show that the solutions constructed in section 3 can be extended beyond the past light cone to infinity. Finally, in section 5 we derive some scaling properties of the shooting parameters. In the appendix we present an alternative rigorous proof of existence.

\section{Local existence}

In the first step we will analyse the behaviour of solutions near the boundary points $\rho=0$ and $\rho=1$ and prove the local existence of one-parameter families of regular solutions there.

Near $\rho=0$ we introduce a new variable $v=u^{\prime}$ and rewrite equation (5) as the first order system:

$$
\begin{aligned}
& \rho u^{\prime}=\rho v, \\
& \rho v^{\prime}=-2 v+\frac{\rho}{1-\rho^{2}}\left(\frac{4}{p-1} \rho v+\frac{2(p+1)}{(p-1)^{2}} u-u^{p}\right) .
\end{aligned}
$$


This system has the form required by proposition 1 of [8] guaranteeing the existence of a one-parameter family of regular solutions near $\rho=0$ such that

$$
u(\rho)=c+\frac{1}{3}\left(\frac{p+1}{(p-1)^{2}} c-\frac{1}{2} c^{p}\right) \rho^{2}+O\left(\rho^{4}\right) .
$$

Similarly, near $\rho=1$ we put $s=1-\rho, \bar{u}(s)=u(\rho), \bar{v}(s)=\bar{u}^{\prime}(s)$ to get

$$
\begin{aligned}
& s \bar{u}^{\prime}=s \bar{v} \\
& s \bar{v}^{\prime}=\frac{1}{(2-s)}\left(\left(\frac{2}{1-s}-\frac{2(p+1)(1-s)}{p-1}\right) \bar{v}+\frac{2(p+1)}{(p-1)^{2}} \bar{u}-\bar{u}^{p}\right) .
\end{aligned}
$$

In order to bring this into the form required by proposition 1 of [8] we introduce $\tilde{v}=$ $\bar{v}-\frac{p+1}{2(p-1)} \bar{u}+\frac{p-1}{4} \bar{u}^{p}$ and rewrite the system $(8 a)$ and $(8 b)$ as

$$
\begin{aligned}
& s \bar{u}^{\prime}=s\left(\tilde{v}+\frac{p+1}{2(p-1)} \bar{u}-\frac{p-1}{4} \bar{u}^{p}\right), \\
& s \tilde{v}^{\prime}=-\frac{2}{p-1} \tilde{v}+\operatorname{sf}(\bar{u}, \tilde{v}, s),
\end{aligned}
$$

where the function $f(\bar{u}, \tilde{v}, s)$ is analytic in $\bar{u}, \tilde{v}$ and $s$ near $s=0$. We conclude that there is a one-parameter family of regular solutions near $\rho=1$ such that

$$
u(\rho)=b+\frac{1}{2}\left(\frac{1}{2}(p-1) b^{p}-\frac{p+1}{p-1} b\right)(\rho-1)+O\left((\rho-1)^{2}\right) .
$$

The singular solutions behave like $u \sim(1-\rho)^{\frac{p-3}{p-1}}$ near $\rho=1$ leading to a singularity of $u^{\prime}$. For later use it is convenient to introduce the variables $\sigma=(1-\rho)^{2 /(p-1)}, \psi(\sigma)=u(\rho)$ and $\theta(\sigma)=\sigma u^{\prime}$. System $(8 a)$ and $(8 b)$ may then be written in the form

$$
\begin{aligned}
& \psi^{\prime}=-\frac{p-1}{2} \sigma^{\frac{p-5}{2}} \theta, \\
& \theta^{\prime}=g(\psi, \theta, \sigma),
\end{aligned}
$$

where the function $g(\psi, \theta, \sigma)$ is analytic in $\psi, \theta$ and $\sigma$ near $\sigma=0$. This implies that there is a two-parameter family of regular solutions parametrized by the values $b=\psi(0)=u(1)$ and $d=\theta(0)$. These solutions are analytic as functions of $b, d$ and $\sigma$ in a neighbourhood of $\sigma=0$. Thus, the general solution is in fact regular at $\rho=1$ as a function of $\sigma=(1-\rho)^{2 /(p-1)}$ with a convergent Taylor series at $\sigma=0$.

\section{Global behaviour and proof of existence}

The main result of this paper is as follows.

Theorem 1. For any odd $p \geqslant 7$ and any nonnegative integer $n$, equation (5) on the interval $0 \leqslant \rho \leqslant 1$ has an analytic solution $u_{n}(\rho)$ which satisfies the boundary conditions (7) and (10) and for which the function $w_{n}=u_{n} / u_{\infty}-1$, with $u_{\infty}$ defined by (18), has exactly $n+1$ zeros.

Remark. In this section we give a 'physicist' proof based on scaling arguments. We believe that by sacrificing slightly the mathematical rigour we gain better understandability of the basic mechanism which is responsible for the existence and structure of solutions. An alternative rigorous proof is presented in the appendix. 
Proof. First, we need to show that solutions which are regular at $\rho=0$ or $\rho=1$ remain bounded on the whole interval $0<\rho<1$. This is conveniently proven using a suitable Lyapunov function [9] defined as

$$
H=\left(1-\rho^{2}\right) \frac{u^{\prime 2}}{2}+\frac{u^{p+1}}{p+1}-\frac{(p+1) u^{2}}{(p-1)^{2}},
$$

obeying

$H(\rho) \geqslant-\frac{1}{p-1}\left(\frac{2(p+1)}{(p-1)^{2}}\right)^{\frac{2}{p-1}} \quad$ and $\quad H^{\prime}=\left(\frac{p+3}{p-1} \rho-\frac{2}{\rho}\right) u^{\prime 2} \leqslant 0$.

Hence, for solutions regular at $\rho=0$ we get

$$
H(\rho) \leqslant H(0)=\frac{c^{p+1}}{p+1}-\frac{(p+1) c^{2}}{(p-1)^{2}},
$$

which implies

$$
\sqrt{1-\rho^{2}}\left|u^{\prime}(\rho)\right| \leqslant c^{\frac{p+1}{2}}
$$

and

$$
|u(\rho)| \leqslant c \quad \text { for } \quad c \geqslant\left(2(p+1) /(p-1)^{2}\right)^{1 /(p-1)} .
$$

Furthermore, $H(\rho)$ and thus $u(\rho)$ have a finite limit at $\rho=1$.

On the other hand, for solutions regular at $\rho=1$ we may integrate the function $H$ to the left. It is easy to see that

$$
-(H+1)^{-1} H^{\prime} \leqslant 2 / \rho,
$$

hence $H$ stays finite on $0<\rho \leqslant 1$ and with it $u$ and $u^{\prime}$. We emphasize that this argument does not exclude solutions which are regular at $\rho=1$ and singular at $\rho=0$. Actually, such an explicit singular solution

$$
u_{\infty}(\rho)=b_{\infty} \rho^{-\frac{2}{p-1}}, \quad b_{\infty}=\left(\frac{2(p-3)}{(p-1)^{2}}\right)^{\frac{1}{p-1}},
$$

will play an important role in our analysis. Note that $u_{\infty}$ corresponds to the singular static solution $\Phi=b_{\infty} r^{-\frac{2}{p-1}}$ of equation (1).

Next, we consider the behaviour of solutions regular at $\rho=0$, i.e. solutions satisfying the initial condition (7), with large values of $c=u(0)$. To this order we rescale the variables

$$
\rho=\frac{x}{c^{(p-1) / 2}}, \quad u(\rho)=c U(x)
$$

From equation (5) we get

$$
\frac{\mathrm{d}^{2} U}{\mathrm{~d} x^{2}}+\frac{2}{x} \frac{\mathrm{d} U}{\mathrm{~d} x}+U^{p}=\frac{1}{c^{p-1}}\left(x^{2} \frac{\mathrm{d}^{2} U}{\mathrm{~d} x^{2}}+\frac{2(p+1)}{p-1} x \frac{\mathrm{d} U}{\mathrm{~d} x}+\frac{2(p+1)}{(p-1)^{2}} U\right) .
$$

In the limit $c \rightarrow \infty$ we obtain the simple equation

$$
\frac{\mathrm{d}^{2} U}{\mathrm{~d} x^{2}}+\frac{2}{x} \frac{\mathrm{d} U}{\mathrm{~d} x}+U^{p}=0
$$

on the interval $0 \leqslant x<\infty$ together with the boundary condition at $x=0$

$$
U(x)=1-x^{2} / 6+O\left(x^{4}\right) .
$$


Note that the special solution $u_{\infty}$ is invariant under the performed rescaling and thus $U_{\infty}(x)=b_{\infty} x^{-2 /(p-1)}$ solves equation (21). From our numerical analysis we know that $U$ oscillates around $U_{\infty}$ suggesting the change in variables

$$
\bar{U}(\tau)=x^{2 /(p-1)} U(x), \quad \tau=\ln (x) .
$$

Substituting (23) into equation (21) we get the autonomous equation

$$
\frac{\mathrm{d}^{2} \bar{U}}{\mathrm{~d} \tau^{2}}+\frac{p-5}{p-1} \frac{\mathrm{d} \bar{U}}{\mathrm{~d} \tau}+\bar{U}^{p}-\frac{2(p-3)}{(p-1)^{2}} \bar{U}=0 .
$$

Using the Lyapunov function

$h=\frac{1}{2} \frac{\mathrm{d} \bar{U}^{2}}{\mathrm{~d} \tau}+\frac{\bar{U}^{p+1}}{p+1}-\frac{(p-3) \bar{U}^{2}}{(p-1)^{2}} \quad$ with $\quad \frac{\mathrm{d} h}{\mathrm{~d} \tau}=-\frac{p-5}{p-1}\left(\frac{\mathrm{d} \bar{U}}{\mathrm{~d} \tau}\right)^{2}$

we conclude that for $\tau \rightarrow \infty$ the solution $\bar{U}(\tau)$ tends to the fixed point $\bar{U}_{\infty}=b_{\infty}$ of equation (24) and $\frac{\mathrm{d} \bar{U}}{\mathrm{~d} \tau} \rightarrow 0$ for $\tau \rightarrow \infty$. Putting $\bar{U}=b_{\infty}+y$ and $\frac{\mathrm{d} \bar{U}}{\mathrm{~d} \tau}=z$ we find

$$
\begin{aligned}
& \frac{\mathrm{d} y}{\mathrm{~d} \tau}=z, \\
& \frac{\mathrm{d} z}{\mathrm{~d} \tau}=-\frac{p-5}{p-1} z-\frac{2(p-3)}{p-1} y+N(y),
\end{aligned}
$$

where $N(y)$ denotes the nonlinear terms in $y$. Neglecting these nonlinear terms we get a linear system with the eigenvalues $\lambda=\left(-p+5 \pm \mathrm{i} \sqrt{7 p^{2}-22 p-1}\right) / 2(p-1)$. This implies that the solution of equation (21) which is regular at $x=0$ has for $x \rightarrow \infty$ the asymptotic form

$$
U(x)=b_{\infty} x^{-\frac{2}{p-1}}\left(1+A_{0} x^{-\frac{p-5}{2(p-1)}} \sin \left(\omega \ln x+\delta_{0}\right)\right)
$$

with some constants $A_{0}$ and $\delta_{0}$ and $\omega=\sqrt{7 p^{2}-22 p-1} / 2(p-1)$.

In order to prove the existence of regular solutions for sufficiently large $c$ we choose some $x_{0}$, such that for $x>x_{0}$ we can safely use the asymptotic expression (27) for the solution of equation (21) with the boundary condition (22). Furthermore, we choose $c_{0}$ so large that we can neglect the rhs of equation (20) on the interval $0 \leqslant x<x_{0}$ for $c>c_{0}$. Integrating solutions regular at $\rho=0$ to $\rho_{0}=x_{0} / c_{0}^{(p-1) / 2}$ and varying the parameter $c=u(0)$ we get a smooth curve $C_{0}$ in the $\left(u\left(\rho_{0}\right), u^{\prime}\left(\rho_{0}\right)\right)$ plane. Using the asymptotic expression (27) for the part of $C_{0}$ corresponding to $c>c_{0}$ we get

$$
u(\rho) \approx b_{\infty} \rho^{-\frac{2}{p-1}}\left(1+A_{0} c^{\frac{5-p}{4}} \rho^{-\frac{p-5}{2(p-1)}} \sin \left(\omega \ln \left(c^{\frac{p-1}{2}} \rho\right)+\delta_{0}\right)\right) .
$$

Keeping $\rho=\rho_{0} \ll 1$ fixed and letting $c \rightarrow \infty$ (and with it $x$ ) we find that $C_{0}$ spirals down (in the negative direction) to the limit point

$$
P\left(\rho_{0}\right)=\left(b_{\infty} \rho_{0}^{-2 /(p-1)},-\frac{2}{p-1} b_{\infty} \rho_{0}^{-(p+1) /(p-1)}\right) .
$$

We are now ready to show the existence of regular solutions for large $c$. Integrating the solutions regular at $\rho=1$ back to $\rho_{0}$ we obtain a smooth curve $C_{1}$ parametrized by $b$. For $0 \leqslant b \leqslant b_{0}$ this curve goes from the point $(0,0)$ to $\left(b_{0}, 0\right)$ passing through $P\left(\rho_{0}\right)$ for $b=b_{\infty}$. Thus, curve $C_{0}$ intersects curve $C_{1}$ again and again as $c$ goes to infinity (note that curve $C_{1}$ has positive rotation around $\left.P\left(\rho_{0}\right)\right)$. This is illustrated in figure 1 in the case $p=7$. Obviously each such intersection yields a solution which is regular on the whole interval $0 \leqslant \rho \leqslant 1$. As $c$ and thus the domain for $x$ increase the corresponding solutions $U(x)$ perform more and more oscillations around $U_{\infty}(x)$. In fact, each further oscillation of $\bar{U}(x)$ about zero leads 


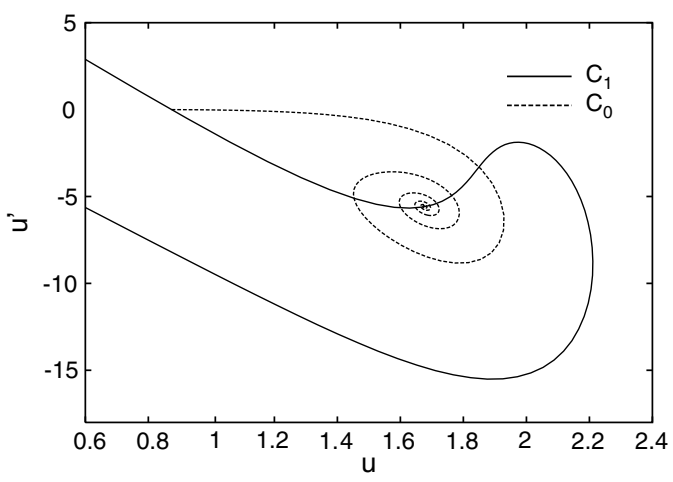

Figure 1. The intersection of curves $C_{0}$ and $C_{1}$ for $\rho_{0}=0.1(p=7)$.

to another cycle in the $\left(u, u^{\prime}\right)$ plane at $\rho_{0}$. Thus, we arrive at the conclusion that for any sufficiently large $n$ there exist regular solutions of equation (5) on the interval $0 \leqslant \rho \leqslant 1$ with $n$ of such oscillations.

In the second step of our existence proof we shall show that we can decrease $n$ and thus prove the existence of regular solutions for any positive integer $n$. The constant solution $u_{0}$ with $c=c_{0}=b_{0}$ has exactly one such intersection. Thus we must be able to decrease the number of intersections to the one decreasing $c$ from some large value $c \gg 1$ corresponding to a regular solution with $n \gg 1$ intersections. Obviously the number of intersections can only change at $\rho=1$, because, by the uniqueness theorem for solutions of differential equations, a double zero of $u(\rho, c)-u_{\infty}(\rho)$ is not possible at a regular point $0<\rho<1$. On the other hand, no solution regular on the whole interval $0 \leqslant \rho \leqslant 1$ can have $b=u(1)=b_{\infty}$. Thus, a change of $n$ can only happen for a value of $c$ corresponding to a solution which is singular at $\rho=1$. We showed in section 2 that such solutions are parametrized by the numbers $b=u(1)$ and $d=V(1)$, where the latter is the finite coefficient of the singular mode. For solutions regular at $\rho=0$ the coefficient $d$ depends smoothly on $c$. Suppose $b(c)=b_{\infty}$ for some $c=c_{s}$. Then, we get a decrease of $n$ exactly by one if either $b-b_{\infty}$ moves from positive to negative values as $c$ decreases through $c_{s}$ and $d\left(c_{s}\right)<0$ or $b-b_{\infty}$ moves from negative to positive values as $c$ decreases through $c_{s}$ and $d\left(c_{s}\right)>0$. This shows that as we decrease $c$ from the value corresponding to a regular solution with $k \gg 1$ intersections to $c_{0}$ we necessarily encounter regular solutions with any value of $n$ between one and $k$. This concludes the proof of theorem 1.

Clearly, there could be more than one regular solution with the same number of intersections, because $b-b_{\infty}$ can have zeros of even order. However, our numerical analysis shows that there is exactly one regular solution for any $n$. The first few solutions generated numerically are shown in figure 2 .

\section{Extension beyond the past light cone}

In this section we show that the solutions constructed above can be smoothly extended beyond $\rho=1$ to infinity.

Consider the function

$$
Q(\rho)=\frac{1}{2}\left(1-\rho^{2}\right) \rho^{3} u^{\prime 2}+\frac{1}{2} \rho^{2}\left(1-\rho^{2}\right) u u^{\prime}-\frac{3 p^{2}-18 p+23}{4(p-1)^{2}} \rho^{3} u^{2}+\frac{1}{p+1} \rho^{3} u^{p+1} .
$$




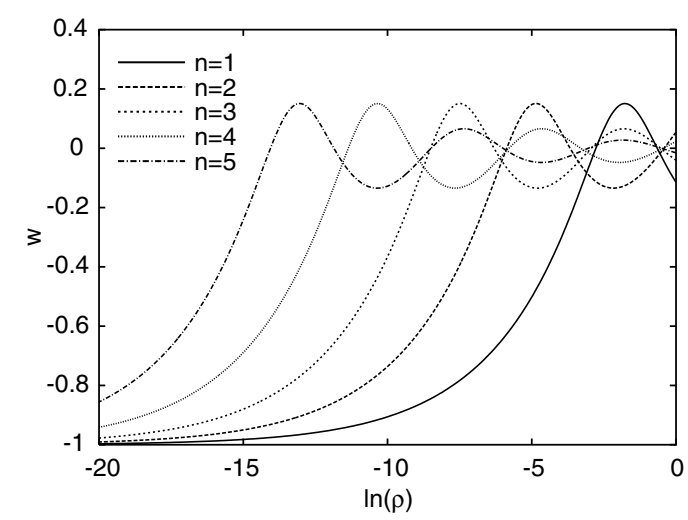

Figure 2. The first five self-similar solutions for $p=7$. To show oscillations around $u_{\infty}$ we plot $w_{n}=u_{n} / u_{\infty}-1$.

This function was introduced by Kavian and Weissler [7] in their study of equation (5). By straightforward (but tedious) computation one gets

$$
Q^{\prime}(\rho)=\frac{5-p}{2(p-1)} \rho^{2}\left[2\left(3 u / 2+\rho u^{\prime}\right)^{2}+\frac{3 p-7}{2(p-1)} u^{2}+\frac{p-1}{p+1} u^{p+1}\right],
$$

hence $Q(\rho)$ is monotone decreasing for $p>5$. Since $Q(0)=0$ for analytic solutions, it follows that $Q(\rho) \leqslant 0$ for $\rho>0$ and thus $u(\rho)$ is positive for $0<\rho \leqslant 1$.

Remark. We note in passing that for $p=5$ it follows from (31) that the function $Q(\rho)$ is the first integral which implies that $u_{0}$ is the only regular self-similar solution.

It is convenient to rewrite equation (5) in a self-adjoint form

$$
\left(\rho^{2}\left(1-\rho^{2}\right)^{\alpha} u^{\prime}\right)^{\prime}=\rho^{2}\left(1-\rho^{2}\right)^{\alpha-1} u\left(b_{0}^{p-1}-u^{p-1}\right) .
$$

Proposition 1. If $u(\rho)$ is a regular solution of equation (32) defined for $0 \leqslant \rho \leqslant 1$ and $u(0)>b_{0}$, then $u(1)<b_{0}$.

Proof. First, note that if $u$ is analytic at $\rho=1$ and $u(1)=b_{0}$, then $u(\rho) \equiv b_{0}$, which contradicts the assumption that $u(0)>b_{0}$. Thus, the case $u(1)=b_{0}$ is impossible. Next, suppose that $u(1)>b_{0}$. If $u(\rho) \geqslant b_{0}$ on the whole interval $0 \leqslant \rho \leqslant 1$ then the rhs of equation (32) is negative and hence by integrating it we get that $u^{\prime}(1)=\infty$. Thus, $u(\rho)<b_{0}$ for some $0<\rho<1$ and therefore $u$ must have a minimum value at some $\rho_{0}$, i.e. $u^{\prime}\left(\rho_{0}\right)=0$ and $0<u\left(\rho_{0}\right)<b_{0}$. If so, between $\rho_{0}$ and 1 there is a $\rho_{1}$ where $u\left(\rho_{1}\right)=b_{0}$ and $u(\rho)<b_{0}$ for $\rho_{0} \leqslant \rho<\rho_{1}$. We now show that this is not possible. To this order we integrate equation (32) over the interval $\rho_{0} \leqslant s<\rho<\rho_{1}$. Since $u^{\prime}\left(\rho_{0}\right)=0$ we get

$$
\rho^{2}\left(1-\rho^{2}\right)^{\alpha} u^{\prime}(\rho)=\int_{\rho_{0}}^{\rho} s^{2}\left(1-s^{2}\right)^{\alpha-1} u\left(b_{0}^{p-1}-u^{p-1}\right) \mathrm{d} s .
$$

We have $u(s)<u(\rho)<b_{0}$; hence $u\left(b_{0}^{p-1}-u^{p-1}\right)=u\left(b_{0}^{p-2}+b_{0}^{p-3} u+\cdots+u^{p-2}\right)\left(b_{0}-u\right)<$ $(p-1) b_{0}^{p-1}\left(b_{0}-u\right)$. Thus,

$$
\begin{gathered}
\int_{\rho_{0}}^{\rho} s^{2}\left(1-s^{2}\right)^{\alpha-1} u\left(b_{0}^{p-1}-u^{p-1}\right) \mathrm{d} s<(p-1) b_{0}^{p-1}\left(b_{0}-u(\rho)\right) \int_{\rho_{0}}^{\rho} s^{2}\left(1-s^{2}\right)^{\alpha-1} \mathrm{~d} s \\
=f(\rho)\left(b_{0}-u(\rho)\right)
\end{gathered}
$$


for some bounded continuous function $f(\rho)$. It follows from (33) and (34) that

$$
\frac{u^{\prime}(\rho)}{b_{0}-u(\rho)}<\frac{f(\rho)}{\rho^{2}\left(1-\rho^{2}\right)^{\alpha}}=g(\rho),
$$

where $g(\rho)$ is a bounded continuous function on $\rho_{0}<\rho \leqslant \rho_{1}<1$. Integrating (35) from $\rho_{0}$ to $\rho$, we get $\ln \left(b_{0}-u\left(\rho_{0}\right)\right)-\ln \left(b_{0}-u(\rho)\right)<\int_{\rho_{0}}^{\rho} g(s) \mathrm{d} s$; hence $\ln \left(b_{0}-u(\rho)\right)$ is bounded for $\rho \leqslant \rho_{1}$. This contradicts the assumption that $u\left(\rho_{1}\right)=b_{0}$ and concludes the proof.

Next, we consider the behaviour of solutions for $\rho>1$.

Proposition 2. If $u(\rho)$ is a solution to equation (32) that is analytic at $\rho=1$ and $u(1)=b<b_{0}$, then $u(\rho)$ is defined for all $\rho>1$ and $0<u(\rho)<b_{0}$.

Proof. Integrating equation (32) from 1 to some $\rho>1$ we get

$$
\rho^{2}\left(\rho^{2}-1\right)^{\alpha} u^{\prime}(\rho)=\int_{1}^{\rho} s^{2}\left(1-s^{2}\right)^{\alpha} u\left(b_{0}^{p-1}-u^{p-1}\right) \mathrm{d} s,
$$

hence if $u$ is bounded, so is $u^{\prime}$ and therefore the solution cannot become singular. Thus, it suffices to show that $0<u(\rho)<b$. From equation (32) we have that $u^{\prime}(\rho)=0$ and $0<u(\rho)<b_{0}$ for $\rho>1$ implies that $u^{\prime \prime}(\rho)<0$; thus $u$ is monotone decreasing. To show that $u$ cannot become negative we introduce the function $f(\rho)=\rho u^{\prime}(\rho)+(\alpha+1) u(\rho) / 2$. Evaluating $f^{\prime}$ at $f=0$ we obtain

$$
\left.f^{\prime}\right|_{f=0}=\frac{1}{2}\left(1-\alpha^{2}\right) u+\frac{2 \rho^{2}}{\rho^{2}-1} u^{p}>0,
$$

which, together with $f(1)=b^{p} / 2 \alpha>0$, implies that $f(\rho)>0$ for $\rho>1$. Since $u^{\prime}(\rho)<0$ (as noted above), we conclude that $u>0$, as claimed.

\section{Large $n$ asymptotics}

Our numerical analysis shows that the shooting parameters $b_{n}$ and $c_{n}$ exhibit remarkable scaling properties in the large $n$ limit. In this section we explain this phenomenon.

In order to derive the large $n$ asymptotic behaviour of solutions which are regular at $\rho=1$ we introduce a new variable $w$ defined by $u=u_{\infty}(1+w)$. Using equations (5) and (10) we find that $w$ satisfies the equation

$\rho^{2}\left(1-\rho^{2}\right) w^{\prime \prime}+\left(\frac{2(p-3)}{p-1} \rho-2 \rho^{3}\right) w^{\prime}-\frac{2(p-3)}{(p-1)^{2}}(1+w)\left((1+w)^{p-1}-1\right)=0$,

with the boundary condition at $\rho=1$

$$
w(\rho)=\frac{b-b_{\infty}}{b_{\infty}}+\frac{p-1}{4} \frac{b}{b_{\infty}}\left(b^{p-1}-b_{\infty}^{p-1}\right)(\rho-1)+O\left((\rho-1)^{2}\right) .
$$

For any $0<\rho_{0} \ll 1$ and $b$ sufficiently close to $b_{\infty}$, the solution of equation (38) satisfying the initial condition (39) will stay arbitrarily close to $w=0$ on the interval $\rho_{0} \leqslant \rho \leqslant 1$; hence it can be approximated by $w=\frac{b-b_{\infty}}{b_{\infty}} w_{L}$, where $w_{L}$ satisfies the linearized equation

$$
\rho^{2}\left(1-\rho^{2}\right) w_{L}^{\prime \prime}+\left(\frac{2(p-3)}{p-1} \rho-2 \rho^{3}\right) w_{L}^{\prime}-\frac{2(p-3)}{(p-1)} w_{L}=0,
$$

with the boundary condition $w_{L}(1)=1, w_{L}^{\prime}(1)=(p-3) / 2$. We could express $w_{L}$ in terms of a hypergeometric function but for our purposes it is sufficient to have its asymptotics for small values of $\rho$

$w_{L} \approx \rho^{\frac{5-p}{2(p-1)}} A_{1} \sin \left(\omega \ln \rho+\delta_{1}\right), \quad \omega=\sqrt{7 p^{2}-22 p-1} / 2(p-1)$, 
Table 1. Shooting parameters of solutions $u_{n}$ and their quotients $\Delta c_{n}=c_{n+1} / c_{n}$ and $\Delta b_{n}=$ $\left(b_{n+1}-b_{\infty}\right) /\left(b_{\infty}-b_{n}\right)$ for $p=7$. The last row for $n=\infty$ corresponds to the analytic results (45) and (46).

\begin{tabular}{|c|c|c|c|c|}
\hline$n$ & $c_{n}$ & $b_{n}$ & $\Delta c_{n}$ & $\Delta b_{n}$ \\
\hline 1 & 2.054390385 & 0.688698572 & 2.8018 & 0.4713 \\
\hline 2 & 5.756037116 & 0.820493408 & 2.4090 & 0.7428 \\
\hline 3 & 13.86655615 & 0.746908360 & 2.5899 & 0.5670 \\
\hline 4 & 35.91343330 & 0.796055093 & 2.4577 & 0.6752 \\
\hline 5 & 88.26661166 & 0.766263419 & 2.5326 & 0.6059 \\
\hline 6 & 223.5507381 & 0.785548198 & 2.4823 & 0.6493 \\
\hline 7 & 554.9215495 & 0.773546658 & 2.5128 & 0.6217 \\
\hline 8 & 1394.439242 & 0.781209584 & 2.4930 & 0.6391 \\
\hline 9 & 3476.402010 & 0.776393971 & 2.5053 & 0.6281 \\
\hline 10 & 8709.676250 & 0.779451184 & 2.4974 & 0.6351 \\
\hline 11 & 21752.40861 & 0.777522645 & 2.5012 & 0.6307 \\
\hline 12 & 54434.14714 & 0.778744142 & 2.4993 & 0.6334 \\
\hline 13 & 136047.6759 & 0.777972446 & 2.5000 & 0.6317 \\
\hline 14 & 340293.1022 & 0.778460765 & 2.5008 & 0.6328 \\
\hline 15 & 850746.1358 & 0.778152079 & 2.5003 & 0.6321 \\
\hline$\infty$ & $\infty$ & 0.778271716 & 2.5005 & 0.6324 \\
\hline
\end{tabular}

where $A_{1}$ and $\delta_{1}$ are constants. Thus, the solution regular at $\rho=1$ with $b$ near $b_{\infty}$ has the following form for small $\rho$

$$
u(\rho) \approx u_{\infty}\left(1+\frac{b-b_{\infty}}{b_{\infty}} \rho^{\frac{5-p}{2(p-1)}} A_{1} \sin \left(\omega \ln \rho+\delta_{1}\right)\right) .
$$

The key point is that the regions of validity of the approximations (42) and (28) overlap so we can match them. Matching the amplitudes of solutions (28) and (42) at $\rho_{0}$ we get the condition

$$
A_{0} c^{\frac{p-5}{4}}= \pm A_{1} \frac{b-b_{\infty}}{b_{\infty}} .
$$

On the other hand, using (28) and the fact that the phases of two adjacent solutions $u_{n}(\rho)$ and $u_{n+1}(\rho)$ differ by $\pi$ we obtain

$$
\frac{p-1}{2} \omega \ln \left(\frac{c_{n+1}}{c_{n}}\right)=\pi
$$

hence

$$
\frac{c_{n+1}}{c_{n}} \approx \mathrm{e}^{\frac{2 \pi}{(p-1) \omega}}
$$

which, using (43), implies that

$$
\frac{b_{n+1}-b_{\infty}}{b_{\infty}-b_{n}} \approx \mathrm{e}^{-\frac{(p-5) \pi}{2(p-1) \omega}}
$$

The numerical verification of these scaling properties is shown in table 1 in the case $p=7$.

Note added in proof. $\mathrm{P}$ Breitenlohner (personal communication) pointed out that a minor modification of our proof applies also to even values of $p$; hence the conclusion of theorem 1 holds for all integers $p \geqslant 6$. 


\section{Acknowledgments}

We are grateful to Peter Breitelohner for the careful reading of the manuscript and comments which helped us to correct and improve it. We thank the referee for pointing out [10] where similar ideas to that of section 3 were used before to prove the existence of self-similar solutions of a nonlinear heat equation. PB and AW thank the Mathematisches Forschungsinstitut in Oberwolfach for supporting this project under the 'Research in Pairs' program. The work of PB was supported in part by the Polish Ministry of Science Grant No 1PO3B01229.

\section{Appendix}

We present here an alternative version of the proof of theorem 1 . Throughout the appendix the range of $\rho$ is $0 \leqslant \rho \leqslant 1$.

We know from section 2 and equation (16) that given any $c$ there is a unique solution $u(\rho, c)$ of equation (5) satisfying $u(0, c)=c$ defined for all $0 \leqslant \rho \leqslant 1$. For $\rho>0$ we define $v(\rho, c)=u(\rho, c) / u_{\infty}(\rho)$ and $w(\rho, c)=v(\rho, c)-1$. We also define the function

$$
H_{v}=\frac{1}{2} \rho^{2}\left(1-\rho^{2}\right) v^{\prime 2}-\alpha(1-\alpha)\left(\frac{v^{2}}{2}-\frac{v^{p+1}}{p+1}\right) .
$$

Using equation (5) we get

$$
H_{v}^{\prime}=\frac{5-p}{p-1} \rho v^{\prime 2}
$$

hence $H_{v}$ is decreasing along solutions. Furthermore, $H_{v}$ has a minimum equal to $-(p-3) /\left(p^{2}-1\right)$ for $v^{\prime}=0$ and $v=1$.

Lemma 1. Let $d=(p-1) / p$. If $c>b_{0} / d$, then there is $\rho_{1}=\rho_{1}(c)<\left(b_{\infty} / d c\right)^{1 / \alpha}$ such that $w\left(\rho_{1}, c\right)=0$ and $0<\rho_{1} w^{\prime}\left(\rho_{1}, c\right)<\alpha$.

Proof. It follows from equation (32) that $u(\rho, c)$ is a decreasing function of $\rho$ for $c>b_{0}$ as long as $u>b_{0}$. Integrating equation (32) and noting that $0<u(\rho, c) \leqslant c$ we get

$$
\begin{aligned}
\rho^{2}\left(1-\rho^{2}\right)^{\alpha} u^{\prime}(\rho) & =-\int_{0}^{\rho} s^{2}\left(1-s^{2}\right)^{\alpha-1}\left[u(s)^{p}-\alpha(1+\alpha) u(s)\right] \mathrm{d} s> \\
& -\int_{0}^{\rho} s^{2}\left(1-s^{2}\right)^{\alpha-1} u(s)^{p} \mathrm{~d} s>-\int_{0}^{\rho} s^{2}\left(1-s^{2}\right)^{\alpha-1} c^{p} \mathrm{~d} s .
\end{aligned}
$$

Thus

$\rho^{2}\left(1-\rho^{2}\right)^{\alpha} u^{\prime}(\rho)>-\int_{0}^{\rho} \frac{s^{2}\left(1-s^{2}\right)^{\alpha-1}}{\left(1-\rho^{2}\right)^{\alpha-1}} c^{p} \mathrm{~d} s>-\int_{0}^{\rho} s^{2} c^{p} \mathrm{~d} s=-\frac{\rho^{3}}{3} c^{p}$,

hence

$$
u^{\prime}(\rho)>-\frac{\rho}{3\left(1-\rho^{2}\right)} c^{p} .
$$

Integrating (51) from 0 to $x$ where $u(x, c)=d c$ gives

$$
-c(1-d)>\frac{c^{p}}{6} \ln \left(1-x^{2}\right), \quad \text { so } x^{2}>1-\exp \left(-\frac{6}{p c^{p-1}}\right)>\frac{6}{p c^{p-1}} \text {. }
$$

Let $y$ be a point where $u_{\infty}(y)=b_{\infty} y^{-\alpha}=d c$, that is, $y=\left(b_{\infty} / d c\right)^{1 / \alpha}$. We have

$$
\left(\frac{b_{\infty}}{d}\right)^{2 / \alpha}=\left(\frac{b_{\infty}}{d}\right)^{p-1}=\left(\frac{p}{p-1}\right)^{p-1} \frac{2(p-3)}{(p-1)^{2}}<\frac{6}{p}
$$


hence $x>y$, and therefore $u(y, c)>u(x, c)=d c=u_{\infty}(y)$ or $w(y, c)>0$. Since $w(\rho, c)<0$ for small $\rho$, we conclude that there is a $\rho$ such that $w(\rho, c)=0$ and if $\rho_{1}$ is the smallest such $\rho$, then $w^{\prime}\left(\rho_{1}, c\right)>0$. Note that $\rho_{1}<y$. This concludes the proof of lemma 1 .

Corollary 1. $w(\rho)>-2 \alpha$ for $\rho>\rho_{1}$.

Proof. Using the fact that the function $H_{v}(\rho)$ is monotone decreasing we obtain

$$
H_{v}(\rho) \leqslant H_{v}\left(\rho_{1}\right) \leqslant \frac{\alpha^{2}}{2}-\alpha(1-\alpha)\left(\frac{1}{2}-\frac{1}{p+1}\right),
$$

for $\rho \geqslant \rho_{1}$. On the other hand, for $\rho \geqslant \rho_{1}$

$H_{v}(\rho)=\frac{1}{2} \rho^{2}\left(1-\rho^{2}\right) v^{\prime 2}-\alpha(1-\alpha)\left(\frac{v^{2}}{2}-\frac{v^{p+1}}{p-1}\right) \geqslant-\alpha(1-\alpha)\left(\frac{v^{2}}{2}-\frac{v^{p+1}}{p-1}\right)$.

Combining equations (54) and (55) we have

$f(v) \equiv \frac{\alpha^{2}}{2}-\alpha(1-\alpha)\left(\frac{1}{2}-\frac{1}{p+1}\right)+\alpha(1-\alpha)\left(\frac{v^{2}}{2}-\frac{v^{p+1}}{p-1}\right) \geqslant 0$,

for $\rho \geqslant \rho_{1}$. Note that $f^{\prime}(v)>0$ for $v<1$. A straightforward calculation yields $f(1-2 \alpha)<0$ if $p \geqslant 7$; hence $v>1-2 \alpha$ (or $w>-2 \alpha$ ) for $\rho \geqslant \rho_{1}$, as claimed.

Let us define

$$
R(\rho, c)=\sqrt{w(\rho, c)^{2}+w^{\prime}(\rho, c)^{2}}, \quad 0<\rho \leqslant 1 .
$$

By uniqueness of solutions of ODEs, a solution starting at $\left(u, u^{\prime}\right)=(c, 0)$ at $\rho=0$ cannot have $\left(u(\rho, c), u^{\prime}(\rho, c)=\left(u_{\infty}(\rho), u_{\infty}^{\prime}(\rho)\right)\right.$, which implies that $R(\rho, c)>0$. Thus, we may also define

$$
\Theta(\rho, c)=\arctan \left(\frac{\rho w^{\prime}(\rho, c)}{w(\rho, c)}\right) \quad \text { up to a multiple of } \pi .
$$

Since the region $\{(\rho, c) \mid 0<\rho \leqslant 1, c \geqslant 0\}$ is simply connected we may unambiguously define a function $\Theta(\rho, c)$ once we specify its value at any point in the domain. Since $w(\rho, 0) \equiv-1, w^{\prime}(\rho, 0) \equiv 0$, we set $\Theta(1 / 2,0)=\pi$. Note that $\lim _{\rho \rightarrow 0+} w(\rho, c)=-1$ and $\lim _{\rho \rightarrow 0+} \rho w^{\prime}(\rho, c)=0$; hence $\lim _{\rho \rightarrow 0+} \Theta(\rho, c)=\pi$.

Lemma 2. Let $\rho_{2} \ll 1$. We have $\lim _{c \rightarrow \infty} \Theta\left(\rho_{2}, c\right)=-\infty$.

Proof. Consider the equation satisfied by the function $\Theta(\rho)=\Theta(\rho, c)$ :

$$
\begin{aligned}
\Theta^{\prime}(\rho)=-\frac{1}{\rho}[ & \sin ^{2} \Theta+\frac{(p-5)-\rho^{2}(p-1)}{(p-1)\left(1-\rho^{2}\right)} \sin \Theta \cos \Theta \\
& \left.+\frac{2(p-3) v}{(p-1)^{2}\left(1-\rho^{2}\right)}\left(1+v+\cdots+v^{p-2}\right) \cos ^{2} \Theta .\right]
\end{aligned}
$$

We will show that the quantity in brackets is bounded from below by a positive constant $\eta$ for $\rho \geqslant \rho_{1}$. It follows that

$$
\begin{aligned}
\lim _{c \rightarrow \infty} \Theta\left(\rho_{2}, c\right) & =\lim _{c \rightarrow \infty}\left(\Theta\left(\rho_{1}(c), c\right)+\int_{\rho_{1}(c)}^{\rho_{2}} \Theta^{\prime}(\rho) d \rho\right) \leqslant \frac{\pi}{2} \\
& +\lim _{c \rightarrow \infty} \int_{\rho_{1}(c)}^{\rho_{2}}\left(-\frac{\eta}{\rho}\right)=\frac{\pi}{2}-\eta \lim _{c \rightarrow \infty} \ln \frac{\rho_{2}}{\rho_{1}(c)}=-\infty,
\end{aligned}
$$

and the lemma is proven. 
To get a bound on the quantity in brackets we regard it as a (formal) quadratic form in $\sin \Theta$ and $\cos \Theta:[]=A \sin ^{2} \Theta+B \sin \Theta \cos \Theta+C \cos ^{2} \Theta$, where

$A=1, \quad B=\frac{(p-5)-\rho^{2}(p-1)}{(p-1)\left(1-\rho^{2}\right)}, \quad C=\frac{2(p-3) v}{(p-1)^{2}\left(1-\rho^{2}\right)}\left(1+v+\cdots+v^{p-2}\right)$.

Since $\rho<\rho_{2}$, we may replace these coefficients by

$A=1, \quad \tilde{B}=\frac{(p-5)}{(p-1)}, \quad \tilde{C}=\frac{2(p-3) v}{(p-1)^{2}}\left(1+v+\cdots+v^{p-2}\right)$.

We need to show that the discriminant $\Delta=\tilde{B}^{2}-4 A \tilde{C}$ is negative for $v>1-2 \alpha$. We have

$$
\Delta=\frac{(p-5)^{2}}{(p-1)^{2}}-\frac{8(p-3)}{(p-1)^{2}}\left(1+v+\cdots+v^{p-2}\right) .
$$

Clearly, $(p-1)^{2} \Delta$ is a decreasing function of $v$. When $v=1-2 \alpha$ we get

$$
(p-1)^{2} \Delta=\left(2 p^{2}-8 p+6\right)\left(\frac{p-5}{p-1}\right)^{p}-p^{2}+6 p-5
$$

which is negative for $p \geqslant 7$; hence $\Delta<0$ which concludes the proof of lemma 2 .

Next, we consider solutions $U(\rho, b)$ which start from initial value (10). As we showed in section 3 these solutions are defined for $0<\rho \leqslant 1$; hence we may define

$$
\tilde{R}(\rho, b)=\sqrt{W(\rho, b)^{2}+W^{\prime}(\rho, b)^{2}}, \quad 0<\rho \leqslant 1,
$$

where $W(\rho, b)=U(\rho, b) / u_{\infty}(\rho)-1$. By uniqueness of solutions of ODEs, a solution starting at $\rho=1$ cannot have $\left(U(\rho, b), U^{\prime}(\rho, b)=\left(u_{\infty}(\rho), u_{\infty}^{\prime}(\rho)\right)\right.$, unless $b=b_{\infty}$, which implies that $\tilde{R}(\rho, b)>0$ if $b \neq b_{\infty}$. Thus, we may also define

$$
\tilde{\Theta}(\rho, b)=\arctan \left(\frac{\rho W^{\prime}(\rho, b)}{W(\rho, b)}\right) \quad \text { up to a multiple of } \pi \text {. }
$$

Since the region $\left\{(\rho, b) \mid 0<\rho \leqslant 1,0 \leqslant b \leqslant b_{\infty}\right\}$ is simply connected we may unambiguously define a function $\tilde{\Theta}(\rho, b)$ once we specify its value at any point in the domain. We set $\tilde{\Theta}(1,0)=\pi$.

We define a map

$\Phi: R_{+}=\{c \mid c \geqslant 0\} \rightarrow R_{+}^{2}=\left\{(x, y) \in R^{2} \mid y>0\right\}, \quad \Phi(c)=\left(\Theta\left(\rho_{0}, c\right), R\left(\rho_{0}, c\right)\right)$

and a map

$\Psi_{n}: t \in\left(-\infty, b_{\infty}\right) \rightarrow R_{+}^{2}, \quad \Psi_{n}(t)= \begin{cases}\left(\tilde{\Theta}\left(\rho_{0}, b\right)-2 n \pi\right. & \text { if } 0 \leqslant t<b_{\infty}, \\ \left.\tilde{R}\left(\rho_{0}, b\right)\right) & \text { (segment } 1), \\ (\pi-2 n \pi, 1-t) & \text { if } t<0\end{cases}$

Note that if $\Psi_{n}(b)=\Phi(c)$ for some $b \in\left(0, b_{\infty}\right)$ and some $c$, then $\Phi(c)$ cannot lie on segment 2 because $w\left(\rho_{0}, c\right) \geqslant-1$ and $y \cos x=-y<-1$ on segment 2. Thus, if $\Psi_{n}(b)=\Phi(c)$ for some $b \in\left(0, b_{\infty}\right)$ and some $c$, then the functions $u(\rho, c)$ and $U(\rho, b)$ and their derivatives match at $\rho_{0}$; hence we have a solution defined on the whole interval $0 \leqslant \rho \leqslant 1$ in the $n$th 
nodal class. The integer part of $(\tilde{\Theta}(1, b)-\Theta(0, c)) / \pi$ counts the number of zeros of $w$. In particular, $\Psi_{0}(0)=\Phi(0)$ since $u(\rho, 0) \equiv U(\rho, 0) \equiv 0$ is a solution in the 0 th nodal class.

Remark. The choices of $\Theta(1 / 2,0)=\pi$ and $\tilde{\Theta}(1,0)=\pi$ were made so that the indexing of solutions is correct.

Now we are ready to prove the following proposition.

Proposition 3. For any positive integer $n$ there is $B_{n}<b_{\infty}$ such that the solution $U\left(\rho, B_{n}\right)$ is in the $n$th nodal class.

Proof. Since $b_{\infty}<b_{0}$, there is a $\rho_{0}<1$ such that $U\left(\rho_{0}, b\right)<b_{0}$ for all $b \in\left[0, b_{\infty}\right]$. Note that $U^{\prime}(1, b)<0$ for $b \leqslant b_{\infty}$ and thus $U^{\prime}(\rho, b)<0$ on $\rho_{0}<\rho<1$ because $U^{\prime \prime}(\rho, b)>0$ if $U^{\prime}(\rho, b)=0$ and $U(\rho, b)<b_{0}$. It follows that $U(\rho, b) \geqslant 0$ and hence $W(\rho, b) \geqslant-1$ for $\rho_{0}<\rho \leqslant 1$ and $0 \leqslant b \leqslant b_{\infty}$. From this conclude that the curve $\Psi_{n}(b)$ is simple, i.e. $\Psi_{n}(b)=\Psi_{n}(\bar{b})$ implies that $b=\bar{b}$. For nonnegative $b$ and $\bar{b}$ this follows from the uniqueness of solutions of ODEs, while for $\bar{b}<0 \leqslant b, \Psi_{n}(\bar{b})=\Psi_{n}(b)$ is impossible because $W\left(\rho_{0}, b\right)>-1$ by choice of $\rho_{0}$ and $y \cos x=-y<-1$ on segment 2 of the curve. Since $\lim _{b \rightarrow b_{\infty}} \tilde{R}\left(\rho_{0}, b\right)=\tilde{R}\left(\rho_{0}, b_{\infty}\right)=0$ and $\lim _{b \rightarrow-\infty} \tilde{R}\left(\rho_{0}, b\right)=\infty$, it follows from the Jordan curve theorem that the curve $\Psi_{n}$ separates the half-plane $R_{+}^{2}$ into two open regions: $R_{+}^{2} \backslash \operatorname{image}\left(\Psi_{n}\right)=A \cup B$. Moreover, points $p$ and $q$ are in the different components $A$ and $B$ iff there is a curve from $p$ to $q$ crossing image $\left(\Psi_{n}\right)$ transversally at exactly one point.

Note that $\tilde{\Theta}(1, b)>\pi / 2$ if $b<b_{\infty}$ and $\tilde{\Theta}^{\prime}(\rho, b)>0$ when $\tilde{\Theta}(\rho, b)=\pi / 2$; hence $\tilde{\Theta}(\rho, b) \geqslant \pi / 2$ for $b<b_{\infty}$. The region

$$
Z=\left\{(x, y) \in R_{+}^{2} \mid x<\pi / 2-2 n \pi\right\}
$$

is connected and does not meet image $\left(\Psi_{n}\right)$ because $\tilde{\Theta}(\rho, b) \geqslant \pi / 2$; hence $Z$ must be contained in $A$ or $B$; suppose that $Z \subset A$. Curve $\Phi$ meets $A$ because $\lim _{c \rightarrow \infty} \Theta(\rho, c)=-\infty$ by lemma 2 and hence curve $\Phi$ meets $Z \subset A$. Note that image $\left(\Psi_{n}\right) \cap \operatorname{image}\left(\Psi_{m}\right)=\emptyset$ if $n \neq m$ by uniqueness of solutions of ODEs; hence $\Phi(0) \notin \operatorname{image}\left(\Psi_{n}\right)$ if $n>0$.

We will now show that $\Phi(0) \in B$. This will complete the proof because the curve $\Phi$ is connected; hence it must cross image $\left(\Psi_{n}\right)$, which means that there is a solution in the $n$th nodal class. To show this we construct a curve in $R_{+}^{2}$ from $\Phi(0)$ to $Z$ that crosses image $\left(\Psi_{n}\right)$ transversally at exactly one point. We denote by $M$ an upper bound of $\tilde{R}\left(\rho_{0}, b\right)$ on $0 \leqslant b \leqslant b_{\infty}$ and define a curve (see figure 3 )

$$
\Gamma(t)= \begin{cases}(\pi, 1+t) & \text { for } 0 \leqslant t \leqslant M \text { (segment } 1) \\ (\pi+M-t, M+1) & \text { for } M \leqslant t \leqslant(2 n+1) \pi \text { (segment } 2) .\end{cases}
$$

Note that segment 1 of $\Gamma$ cannot meet segment 1 of $\Psi_{n}$ because segment 1 of $\Psi_{n}$ has $w>-1$ and segment 1 of $\Gamma$ has $y \cos x<-1$. Also, segment 1 of $\Gamma$ cannot meet segment 2 of $\Psi_{n}$ because they have different first coordinates $\pi-2 \pi<\pi$. Finally, segment 2 of $\Gamma$ cannot meet segment 1 of $\Psi_{n}$ because $\tilde{R}\left(\rho_{0}, b\right) \leqslant M<M=1$. Thus, the only intersection point of $\Gamma$ and $\Psi_{n}$ is at $(\pi-2 n \pi, M+1)$. This completes the proof of proposition 3 .

Proposition 4. For any positive integer $n$ there is $\tilde{B}_{n}>b_{\infty}$ such that the solution $U\left(\rho, b_{n}\right)$ is in the $n$th nodal class. 


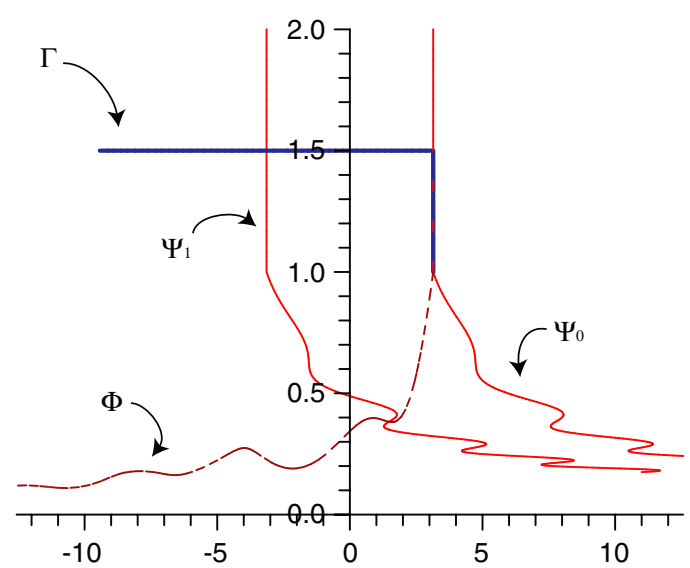

Figure 3. Illustration to the proof of proposition 3 .

(This figure is in colour only in the electronic version)

Proof. The proof proceeds along the same lines as the proof of proposition 3 except for some technical modifications which we leave to the reader.

Theorem 1 is the immediate consequence of propositions 3 and 4 if we set $b_{2 n-1}=B_{n}$ and $b_{2 n}=\tilde{B}_{n}$.

\section{References}

[1] Keller J 1957 Commun. Pure Appl. Math. 10523

[2] John F 1979 Manuscr. Math. 28235

[3] Glassey R 1981 Math. Z. 178233

[4] Dispersive PDE Wiki http://tosio.math.toronto.edu/wiki

[5] Kichenassamy S and Littman W 1993 Commun. Partial Diff. Eqns 181869

[6] Bizoń P, Chmaj T and Tabor Z 2004 Nonlinearity 172187

[7] Kavian O and Weissler F B 1990 Commun. Partial Diff. Eqns 151421

[8] Breitenlohner P, Forgács P and Maison D 1994 Commun. Math. Phys. 163141

[9] Hartman P 1982 Ordinary Differential Equations (New York: Birkhäuser Boston)

[10] Lepin L A 1988 Diff. Eqns 24799 